\title{
LE DEVELOPPEMENT ACTUEL DE LA LIBERTE DE REUNION OU D'ASSOCIATION EN REPUBLIQUE DEMOCRATIQUE DU CONGO (Le défi d'une effectivité).
}

\author{
Par Kapya Kabesa Jean Salem Marcel ${ }^{1}$ et Ntalasha Kisimba ${ }^{2}$
}

\section{INTRODUCTION}

Le développement actuel de la liberté de réunion et d'association en RDC couvre les paradoxes multiples, à savoir, la répression des manifestations publiques, la violence pendant les manifestations pour réclamer ou revendiquer un droit lésé, l'attitude des professionnels des médias vis-à-vis des hommes au pouvoir, la liberté de presse et ses délits, le droit de se réunir, les missions de la police nationale congolaise, bref le respect de l'ordre public, etc.

La liberté d'expression est un droit pour le peuple et constitue l'un des fondements d'une société libre et démocratique, l'une des conditions de son progrès et l'épanouissement de chacun. Pour exercer son pouvoir en pleine conscience, le peuple a droit à être informé complètement sur les divers enjeux politiques, car une démocratie dépend d'un électorat informé, et pour se faire d'une presse libre lui offrant un large éventail d'idées, d'opinions et de points de vue. La plus large liberté d'expression est essentiellement pour mettre en œuvre la responsabilité politique des gouvernants, corollaire de la souveraineté populaire.

Du point de vue standards internationaux, c'est l'article 19 de la déclaration universelle de droits de l'homme et du citoyen de 1789 dispose que « Tout individu a droit à la liberté d'opinion et d'expression, ce qui implique le droit de ne pas être inquiété pour ses opinons et celui de chercher, de recevoir et de reprendre, sans considération de frontières, les informations et les idées par quelque moyen d'expression que ce soit ».

En effet, la doctrine souligne que la liberté de la presse fournit à l'opinion publique l'un des meilleurs moyens de connaître et de juger les idées et les attitudes des dirigeants ${ }^{3}$. A juste titre, DE TOCQUEVILLE explique que le principe du droit à l'information peu importe la façon dont s'exécute et s'exerce; il faut donc organiser l'information pour le peuple. 4

1 Avocat au Barreau de Lubumbashi et Doctorant en Droit à l'Université de Lubumbashi, mail: kabesacaelo@gmail.com

2 Magistrat, Substitut du Procureur de la République et Assistant à la Faculté de Droit de l'Université de Lubumbashi.

3 BORIS LIBOIS, Éthique de l'information, essai sur la déontologie journalistique, Ed. Presse Universitaire de Bruxelles, 1994.p.53.

4 DE TOCQUEVILle A. De la démocratie en Amérique, éd. Union Générale, Paris 1990 p.116. 
L'article 8 de la Loi №96-002 du 22 juin 1996 qui régit l'exercice de la liberté de la presse en République Démocratique du Congo définit la liberté de la presse comme : «le droit d'informer, d'être informé, d'avoir ses opinions, ses sentiments et de les communiquer sans aucune entrave, quel que soit le support utilisé, sous réserve du respect de la loi, de l'ordre public, des droits d'autrui et des bonnes mœurs ».

L'article 212, sans faire allusion à la Haute Autorité des Médias ${ }^{5}$, met en place un Conseil Supérieur de l'Audiovisuel et de la Communication (CSAC) en ces termes :

« Il est institué un Conseil Supérieur de l'Audiovisuel et de la Communication doté de la personnalité juridique.

« Il a pour mission de garantir et d'assurer la liberté et la protection de la presse ainsi que de tous les moyens de communication de masse dans le respect de la Loi.

« Il veille au respect de la déontologie en matière d'information et d'accès équitable des partis politiques, des associations et des citoyens aux moyens officiels d'information et de communication. La composition, les attributions, l'organisation et le fonctionnement du CSAC sont fixés par une Loi organique ».

Par ailleurs écrivit un auteur que les professionnels des médias que ça soit de la presse audio-visuelle ou écrite doivent avoir une attitude conséquente en diffusant les informations : ils doivent savoir que le soleil se lève aujourd'hui, il s'élèvera demain et la vie continue...cela implique que le journaliste doit avoir le sens de la mesure ${ }^{6}$.

Une exigence d'un large débat public que consacre le premier amendement de la constitution américaine pris selon la cour suprême des États-Unis repose sur les principes que la diffusion de l'information la plus large possible à partir des sources diversifiées et antagonistes est essentiel pour le bien-être public qu'une presse libre est une condition de société libre.

Le concile Vatican 2 s'exprime qu'aussi bien, trouve-t-on inhérent à la société humaine le droit à l'information sur le sujet qui intéresse les hommes soit en tant qu'individu, soit en tant que membre d'une société selon la situation de chacun; cependant les bons exercices de ses droits requièrent que la communication soit quant à l'objet toujours véridique et dans le respect de la justice et de la charité complète;qu'elle soit quant au mode honnête conve-

5 La Constitution ne dit pas que cette institution est créée en remplacement de la HAM. Et l'allusion souvent faite à la Commission Electorale Indépendante (CEI) vaut la peine d'être relevé à ce niveau. La Constitution ne dit pas non plus que le CENI qu'elle institue à son article 211 est l'équivalent de la CEI, tout autant dissoute de plano. L'apparente survivance de la CEI tient au fait qu'il est recouru aux archives, méthodes et techniques dont elle a fait usage par le passé afin de parachever le processus électoral qu'elle n'organise plus. Ce processus a été récupéré par le Ministère en charge des affaires intérieures.

6 KAPYA KABESA Jean Salem Marcel, les défis de la liberté de presse en RDC(enjeux pour la ville de Lubumbashi,in annales de Kolwezi, n², juillet 2009,pp.34-47Loi organique ${ }^{\circ}$ 2009-257 du 5 mars 2009 relative à la nomination des présidents des sociétés France Télévisions et Radio France et de la société chargée de l'audiovisuel extérieur de la France. 
nable c'est-à-dire dans l'acquisition et dans la diffusion des nouvelles, elle observe absolument les lois morales,les droits et la dignité de l'homme?

Il est désolant de constater que lorsque le pouvoir s'intéresse aux médias, il préfère entériner son pouvoir de contrôle ${ }^{8}$ plutôt que de réfléchir, par exemple, à la non-systématicité d'un renouvellement de concession attribuée aux chaînes ou encore de corréler cette attribution à quelques obligations d'éthique de codécision, comme la mise en place d'une société de rédacteurs à leur tête. Et quand l'initiative n'est pas inintéressante - prenons le cas des Etats généraux de la presse écrite -, le bilan est tel qu'il révèle le leurre de toute cette affaire.

Concernant la sphère médiatique, un nouvel enjeu apparaît peut-être davantage aujourd'hui, dans la mesure où les citoyens cherchent à rendre l'exercice plus "continu" de la démocratie, en défendant par exemple une conception moins intermittente (ayant lieu tous les cinq ans) de la souveraineté, et donc nécessairement plus délibérative et pluraliste. Par rapport à cette idée d'une continuité renforcée de la démocratie, les médias joueront sans doute un rôle déterminant, soit en consolidant l'espéré paradigme délibératif, soit, à l'inverse, en organisant son simulacre. Les réseaux sociaux et les nouveaux médias - qui démocratisent la donne, hybrident les contenus, donc croisent les savoirs profanes et experts - rendent certes l'offre d'information plurielle mais demeurent encore trop les chambres d'écho des cycles d'engouement et des rumeurs invérifiables. En ce sens, rien n'est moins sûr que le bel avenir d'une information libre, éclairée, gratuite, indépendante et pluraliste.

Si les médias ont un rôle si fondamental dans la démocratie, c'est certes pour défendre le lien irréductible entre la citoyenneté et l'information qualitative et gratuite, mais aussi parce que le pacte social est par essence un pacte avec la parole (d'où le paradoxe »qui a l'information, a le pouvoir »). Oui, la démocratie est le lieu de la démagogie et de la sophistique. Elle partage, d'ailleurs, cette malchance avec d'autres régimes politiques. Mais ce qu'elle ne partage avec aucun d'eux, c'est le fait qu'elle se fonde sur un double engagement qui consiste, pour l'un, à prendre le risque de dire la vérité et, pour l'autre, à accepter de l'entendre, sans porter préjudice. Ces jeux, comme le souligne le philosophe Michel Foucault, sont au cœur de l'espace public démocratique, soit de la vitalité et de la pérennité démocratiques. Ce pacte, avec le courage de la vérité, les médias ont à l'assumer sans réserve"; ce que CHARVIN exprime en disant qu' « une démocratie d'acquiescement où les citoyens ne sont pas des centres d'initiatives, mais des simples homo economicus (producteurs et consommateurs) est une société dont le régime des libertés est menacé ${ }^{10} »$.

7 Vatican II décret sur les moyens de communication, $n^{\circ} 5$.

8 Loi organique $\mathrm{n}^{\circ}$ 2009-257 du 5 mars 2009 relative à la nomination des présidents des sociétés France Télévisions et Radio France et de la société chargée de l'audiovisuel extérieur de la France.

9 Ouvrage : "La Fin du courage" (Fayard, 2010), Cynthia Fleury, chercheur à la Conservation des espèces, restauration et suivi des populations au Muséum national d'histoire naturelle.

10 CHARVIN, R, et SUEUR, J-J, Droits de l'homme et libertés de la personne, LITEC, 5éme éd, 2007, p.193. 
De notre part, la liberté de réunion et d'association en RDC et spécialement dans la ville de Lubumbashi se heurtent aux influences d'ordre politique et administratif d'une part et par ailleurs leur effectivité se trouve au confluent entre la mission de la police dans le rétablissement de l'ordre public aussi que la soumission des professionnels de médias aux aléas des hommes au pouvoir : ce qui justifie l'intitulé de notre dissertation, à savoir, le développement actuel de la liberté de réunion ou d'association en RDC(défis, enjeux et effectivité).

Doit-on se demander ce qui explique l'ineffectivité du droit de se réunir, de manifester ses opinions, de s'associer dans un Etat ou le maintien de l'ordre public assuré par la police d'une part et de surcroit se trouve menacé ceux qui détiennent le pouvoir politique.

\section{Consécration constitutionnelle des libertés publiques par la RDC.}

I.1. Cadre juridique et reconnaissance au niveau interne.

La Constitution de la RDC du 18 février 2006 a mis au sommet le respect des droits et libertés fondamentaux comme principe fondateur de la nouvelle république. Dans son exposé des motifs, on peut lire ce qui suit: "Le constituant tient à réaffirmer l'attachement de la RDC aux droits humains et aux libertés fondamentales tels que proclamés par les instruments juridiques internationaux auxquels elle a adhéré. Aussi a-t-il intégré ces droits et libertés dans le corps même de la Constitution ».Cela signifie qu'une loi qui serait non conforme à ces droits ne doit pas être appliquée.

Dans la manière de garantir l'ordre public entendue de manière judiciaire et administrative, c'est-à-dire la sécurité des personnes et leurs biens, la tranquillité, l'esthétique, la moralité et la lutte contre les troubles sociaux par la violence pendant les réunions ou les manifestations, le rôle et les missions de la police nationale congolaise(PNC) se sont ainsi trouvés complètement redéfinis.

La PNC est désormais un service public apolitique et soumis à l'autorité civile, au service de la Nation toute entière et sa mission est d'assurer la protection des personnes et des biens et le maintien de l'ordre public dans le respect de la constitution et des lois de la République ${ }^{11}$.

Dans son rôle de maintien et de rétablissement de l'ordre public, quels sont les droits Humains que doit respecter la police? Celle-ci doit veiller particulièrement au respect des libertés publiques :liberté de pensée, de conscience et de religion (article 22), liberté d'expression (article 23),le droit à l'information (article 24) et liberté de la presse,liberté de réunion (article 25), liberté de manifestation (article 26), liberté de circulation (article 30), liberté d'association (article 37), liberté syndicale (article 38 et droit de grève droit de grève (article 39),le droit d'adresser une pétition à l'autorité publique (article 27), telle est l'étendue des libertés publiques.

11 Articles 182,183 et 184 de la Constitution. 
Il faut a jouer que l'Inspection Générale (ex Inspection Générale d'Audit) est un organe de contrôle des activités de la Police Nationale qui compte parmi ses missions essentielles, «l'évaluation du respect des Droits Fondamentaux, Droits de l'Homme, et de la protection des libertés individuelles et collectives, dans l'exercice de la fonction de police ${ }^{12} »$.

\section{I.1.1. Régime juridique de la liberté de réunion et la liberté de manifestation.}

a. Cadre juridique national et international

1. Les standards internationaux et régionaux.

Le Pacte International relatif aux droits civils et politiques en son article 21 dispose que « le droit de réunion pacifique est reconnu. L'exercice de ce droit ne peut faire l'objet que des seules restrictions imposées conformément à la loi et qui sont nécessaires dans une société démocratique, dans l'intérêt de la sécurité nationale, de la sûreté publique, de l'ordre public ou pour protéger la santé ou la moralité publiques, ou les droits et les libertés d'autrui. »

Cependant l'article 11 de la Charte Africaine des Droits de l'Homme et des peuples indique que : "Toute personne a le droit de se réunir librement avec d'autres. Ce droit s'exerce sous la seule réserve des restrictions nécessaires édictées par les lois et règlements, notamment dans l'intérêt de la sécurité nationale, de la sûreté d'autrui, de la santé, de la morale ou des droits et libertés des personnes. »

\section{Le cadre national}

C'est la constitution du 18 février 2006 en article 25 qui dispose que " La liberté des réunions pacifiques et sans armes est garantie sous réserve du respect de la loi, de l'ordre public et des bonnes mœurs. »

De même l'article 26 renchérit en indiquant que « La liberté de manifestation est garantie. Toute manifestation sur les voies publiques ou en plein air, impose aux organisateurs d'informer par écrit l'autorité administrative compétente. Nul ne peut être contraint à prendre part à une manifestation. La loi en fixe les mesures d'application.

Par ailleurs le décret-loi 196 du 29 janvier 1999 portant réglementation des manifestations et réunions publiques en son article 1 : «Tous les congolais ont le droit d'organiser des manifestations et des réunions pacifiques et d'y participer individuellement ou collectivement, publiquement ou en privé, dans le respect des lois, de l'ordre public et des bonnes mœurs».

12 Article 2.a du Décret $n^{\circ} 08 / 23$ du 24 septembre 2008 portant organisation et fonctionnement de l'Inspection Générale d'Audit. Dans ce cadre sa fonction est d'enquêter et proposer des sanctions disciplinaires ou saisir la justice, lorsque des violations des droits et.libertés sont commises par des policiers. 
Ce décret prévoit le régime d'organisation de ces libertés. La circulaire du Ministre de l'Intérieur (002/2006 du 29 juin 2006) est un texte qui permet de mieux conformer le décret aux normes constitutionnelles.

Le Décret-loi fait la distinction entre les réunions et manifestations privées et les manifestations et réunions publiques car elles n'obéissent pas aux mêmes règles :

Aux termes de l'article 2 :- Sont considérées comme manifestations notamment, les marches, les défilés; les cortèges, les cérémonies d'accueil, les processions, à caractère politique culturel ou religieux

-Sont considérées comme réunions, tous rassemblements sédentaires d'au moins deux personnes ne comportant aucun mouvement continu de déplacement d'un lieu à un autre »

Et l'Article 3 stipule que: "Sont considérées comme publiques les manifestations et réunions organisées sur la voie publique ou dans les lieux publics ouverts, non clôturés ou celles auxquelles le public est admis ou invité; Sont considérées comme privées les manifestations et réunions organisées en dehors de la voie publique, dans les lieux publics ou privés fermés et clôturés. »

Il faut relever que les manifestations et réunions privées sont totalement libres. Elles ne sont soumises à aucune formalité. Le régime de l'autorisation préalable a été supprimé, il n'est plus conforme aux normes légales en vigueur. Les manifestations et réunions publiques ne sont pas soumises à autorisation mais à une simple déclaration préalable.

Cela doitse faire :- au moins 24 heures à l'avance pour les manifestations sur les voies ou lieux publics ou en plein air, concernant les rassemblements électoraux;

- au moins 3 jours à l' avance pour toute autre rassemblement.

Il s'agit d'une simple information. Il n'est pas nécessaire d'attendre une réponse de l'administration serait-ce sous la forme d'un accusé de réception. Il suffit que l'information ait été faite pour que la manifestation puisse se dérouler. Cette déclaration doit être déposée auprès de l'autorité locale territorialement compétente (Gouverneur de Province, Gouverneur de la ville de Kinshasa, Commissaire du District, Maire de la Ville, Chef de Cité, Chef de Secteur ou Chef de Chefferie)

\section{b. Raison d'être des libertés de réunion ou de manifestation en RDC}

Les libertés d'association, de réunion pacifique et de manifestation sont des droits fondamentaux car ils permettent aux citoyens de se regrouper et d'agir collectivement pour promouvoir et défendre des intérêts et de convictions communes de tous ordres.

Ces libertés sont particulièrement importantes dans les domaines politique et social. En matière politique, elles permettent aux différentes tendances politiques de s'organiser et présenter leurs programmes à la population. En matière sociale car elles permettent l'expression des revendications sociales liées aux conditions de vie de la population et notamment aux conditions de travail.

La liberté syndicale et la liberté politique sont des formes d'expression de ces libertés et elles bénéficient en tant que telles de protections particulières. Il est donc particulièrement 
important que l'exercice de ces libertés qui correspond au droit de participer à la vie politique et sociale du pays ne soit pas restreint illégalement par les forces de sécurité, notamment par les services de police.

\section{c. L'organisation du droit de réunion et de manifestation en RDC}

Le Décret-loi 196 du 29 janvier 1999 portant réglementation des manifestations et réunions publiques, prévoit l'encadrement de ces libertés. Il est antérieur à la constitution et il est le reflet de la période politique qui l'a vu naître. Les dispositions non conformes aux nouveaux standards posés par la Constitution doivent être interprétés au regard du nouveau cadre légal posé par cette dernière et par les engagements internationaux de la RDC.

La loi électorale de 2006 (article 29) et une circulaire du Ministre de l'Intérieur (002/2006 du 29 juin 2006) ont précisé que : «Les dispositions de la Constitution et de la loi électorale (...) consacrent le principe d'information ou de la déclaration préalable et annulent celui de l'autorisation préalable prévu par le décret-loi $\mathrm{n}^{\circ} 196$ du 29 janvier $1999 \ldots$...

A. nécessités de l'intervention liée à l'ordre public

1. impératifs de sécurité

Seuls des impératifs liés à la sécurité ou à l'ordre public, peuvent justifier l'intervention de l'autorité administrative.

La modification de la date et de l'itinéraire d'une manifestation peut ainsi être proposée par les autorités administratives, mais elle ne peut se faire qu'en accord avec les organisateurs de la manifestation. L'autorité administrative n'est pas autorisée à intervenir directement et de façon autoritaire pour modifier les modalités du déroulement d'une manifestation.

La responsabilité première de la sécurité d'une manifestation incombe aux organisateurs du rassemblement, qui doivent disposer d'un service d'ordre ou prendre toutes autres mesures pour éviter des débordements susceptibles de troubler l'ordre public et de porter atteinte aux personnes.

Les autorités publiques saisies de la déclaration préalable doivent veiller au déroulement pacifiques des réunions et manifestations sans tenter de les entraver.

En effet l'article 7 de la circulaire de 2006 indique : « Les autorités compétentes saisies de la déclaration préalable ont l'obligation de veiller au déroulement pacifique des manifestations ou réunions publiques organisées dans leur ressort territorial ainsi qu'au respect de l'ordre public et des bonnes mœurs sans tenter de les entraver. Toutefois, elles peuvent, de commun accord avec les organisateurs ou leurs mandataires, différer la date ou modifier l’itinéraire ou le lieu des manifestations ou réunions publiques envisagées. » 
L'autorité publique ne doit intervenir que si ces mesures s'avèrent inefficaces ce qui indique l'Article 8 de la même circulaire « Les forces de l'ordre n'interviennent pour disperser les manifestants qu'en cas de débordements ou de troubles graves. »

\section{I.1.2. Limites de l'exercice des libertés de réunion et de manifestation?}

La liberté des réunions pacifiques et la liberté de manifestation sont des droits d'ordre constitutionnel, sanctionnés par les traités internationaux. Comme pour toutes les libertés publiques, les seules restrictions à leur exercice ne peuvent être tirées que des atteintes aux droits d'autrui, à l'ordre public et aux bonnes mœurs tel qu'énoncées par la loi (article 25 Constitution, art. 1 Décret-loi 1999).

Comme c'est le cas pour les autres libertés publiques, la notion d'ordre public doit être strictement interprétée, le risque de trouble invoqué devant être sérieux et caractérisé. L'interprétation extensive de cette notion permet souvent aux autorités publiques, souvent sous couvert de sécurité publique, à commettre de graves violations de ces libertés. 125

\section{I.1.3. Les règles d'intervention de la police en cas de trouble à l'ordre public}

Tout déploiement des unités de police dans le cadre des opérations de maintien de l'ordre doit se faire sur demande d'une autorité civile : la police ne peut pas entreprendre de telles actions de sa propre initiative.

Le déclenchement de l'intervention des forces de police pour une opération de maintien de l'ordre obéit, en toute matière et à l'occasion de débordements graves intervenus lorsqu'une réunion ou une manifestation publique, obéit aux règles suivantes.

\section{a. La nécessité d'une réquisition émanant de l'autorité civile}

L'ordre d'intervention doit être donné aux services de police par voie de réquisition : selon la loi organique du 10 août 2011 en son article 75 : «L'action des autorités administratives responsables du maintien et du rétablissement de l'ordre public s'exerce à l'égard de la Police nationale par voie de réquisition. Sauf urgence ou cas de force majeure, toute réquisition doit être écrite. Elle mentionne la disposition légale en vertu de laquelle elle est faite, en indique l'objet, est datée et porte les noms et qualité ainsi que la signature de l'autorité compétente. Toutefois, la réquisition verbale faite en cas d'urgence ou de force majeure doit être confirmée par écrit dans les vingt-quatre heures».

Il existe trois types de réquisition :

- Réquisition générale ayant pour but de rassembler de manière générale des éléments humains et matériels aptes à intervenir s'il y en a besoin; 
- Réquisition particulière ayant pour but de rassembler une unité de police apte à intervenir par la force;

- Réquisition spéciale donnant aussi droit à l'utilisation des armes à feu dans le cadre d'une intervention.

Les précautions à prendre par les services de police, requis d'intervenir pour surveiller le déroulement d'une manifestation.

-Les mesures préventives

Les services de police du maintien de l'ordre agissent d'abord à titre préventif et doivent donc à cet effet faire une analyse correcte de la situation de façon à pouvoir anticiper les difficultés à prévoir et procéder dans la mesure du possible à l'organisation de mesures préventives et au suivi de la manifestation en coordination avec ses organisateurs.

Les responsables de la police doivent donc:

- se renseigner dans la mesure de possible sur les objectifs de la démonstration;

- identifier des difficultés possibles en coordination avec les organisateurs;

- maintenir des contacts réguliers avec les organisateurs dans les jours précédant la démonstration;

- assurer un briefing adéquat des policiers appelés à assurer le bon déroulement de la manifestation;

- suivre le déroulement de la manifestation avec la vigilance et la prudence nécessaire pour prévenir les heurts et éviter la propagation des éventuels désordres en cas d'utilisation de la force et existence d'un trouble grave à l'ordre public.

Le Policier doit toujours garder à l'esprit que les manifestants ont le droit de manifester ou se réunir. Une réquisition de dispersion ne peut être prise que s'il existe un trouble grave à l'ordre public ce qu'exprime l'article 8 du Décret-loi de 1999 précise que « Les Forces de l'ordre n'interviennent pour disperser les manifestations qu'en cas de débordements ou de troubles graves. »

-Les principes encadrant l'usage de la force

Les normes généralement reconnues en matière d'application des lois par des fonctionnaires de la police, se trouvent exprimées dans le « Code de conduite pour les responsables de l'application des lois et les Principes Basiques sur l'utilisation de la force et des armes de feu par les responsables

D'application de la loi » qui a fait l'objet de la résolution 34/169 des Nations-Unies adoptée en 1990 à la Havane. Les principes essentiels relatifs à l'usage de la force sont les suivants :

-Tout fonctionnaire chargé du déroulement des manifestations doit respecter l'application des lois en la matière, et particulièrement les droits fondamentaux des personnes, notamment en ce qui concerne l'usage de la force.

-L'usage de la force, doit être nécessaire à la prévention des troubles. Le principe fondamental qui s'applique à toute intervention de la police est celui de la proportionnalité : les moyens utilisés doivent être proportionnés au trouble qu'il s'agit de faire cesser ou de prévenir. 
-Les règles posées par la loi organique sur l'organisation et fonctionnement de la Police Nationale Congolaise du 11 aout 2011 : la force ne doit être utilisée qu'en cas de nécessité absolue et en respectant les principes de proportionnalité et de progressivité :

Son Article 8 dispose : «La Police nationale ne recourt à la force qu'en cas de nécessité absolue et uniquement pour atteindre un objectif légitime. En tout état de cause, l'usage de la force doit respecter le principe de proportionnalité et de progressivité; l'usage des armes doit être d'une nécessité absolue pour assurer sa défense ou celle d'autrui.

De même, l'article 9 : "Dans l'exercice de leurs fonctions, les agents de la Police peuvent, en cas d'absolue nécessité, employer la force des armes blanches ou des armes à feu : -lorsqu'ils ne peuvent défendre autrement le lieu qu'ils occupent, les établissements, les postes ou les personnes qui leur sont confiées;

-lorsque les violences ou voies de fait sont exercées contre eux-mêmes ou autrui. Sans préjudice des dispositions de l'alinéa ler du présent article, les agents de la Police nationale font usage, en cas d'absolue nécessité, d'armes blanches sans réquisition préalable lorsqu'ils sont chargés, dans l'exercice de leurs fonctions, de disperser des attroupements ou de réprimer des émeutes; mais ils ne peuvent faire usage d'armes à feu que sur réquisition préalable de l'autorité légalement responsable du maintien de l'ordre.

Avant tout usage d'armes à feu, cette autorité fait trois sommations formulées à haute et intelligible voix dans les termes suivants : « obéissance à la loi; on va faire usage d'armes à feu; que les bons citoyens se retirent ».

Ces principes sont d'une très grande importance : Ils permettent aux responsables de prendre les mesures strictement nécessaires au maintien de l'ordre tout en assurant aux citoyens le respect de leurs libertés fondamentales

-Une intervention nécessaire qui respecte les principes de proportionnalité et de la progressivité permet d'éviter qu'une manifestation ne dégénère en violences, et particulièrement en violences commises par des policiers.

\section{I.2. La liberté d'association}

a. de la liberté d'association.

Une association se définit comme une réunion durable de personnes ou d'intérêts en vue d'un but commun.

La liberté d'association, comme les libertés de réunion et de manifestation, est un droit qui permet aux citoyens de s'exprimer collectivement et de participer à la vie sociale. Elle se distingue de ces libertés, en ce sens qu'elle permet des regroupements organisés qui ne sont plus ponctuels, organisés autour d'un évènement mais qui ont une certaine permanence dans le temps de façon à pouvoir développer les activités correspondants à la défense et la promotion de valeurs ou objectifs communs.

Ces valeurs et objectifs communs peuvent être de tous ordres, culturel, religieux, social, économique ou politique. 
La liberté d'association permet à la société civile de participer activement à la vie sociale du pays et de faire entendre les différents points de vue des citoyens qui peuvent manifester leurs préférences politiques, leurs aspirations économiques et sociales, leurs préoccupations dans le domaine des Droits de l'Homme ou de la conservation de la nature, leurs engagements dans le domaine éducatif, etc.

La liberté d'association est un important moyen d'influer sur les choix politiques, économiques et sociaux des gouvernants et ainsi de donner vie et réalité au fonctionnement démocratique.

La liberté d'association est un des moyens d'expression essentiels de la liberté syndicale et de la liberté de culte. La liberté syndicale est particulièrement importante en période d'élections lorsqu'il s'agit pour les travailleurs de faire connaître leurs revendications aux futurs élus et pour l'opposition politique de pouvoir proposer son ou ses programmes aux électeurs.

\section{b. Cadre juridique national et standards internationaux}

Le pacte international relatif aux droits civils et politiques en son Article 22 : « Toute personne a le droit de s'associer librement avec d'autres, y compris le droit de constituer des syndicats et d'y adhérer pour la protection de ses intérêts. L'exercice de ce droit ne peut faire l'objet que des seules restrictions prévues par la loi et qui sont nécessaires dans une société démocratique, dans l'intérêt de la sécurité nationale, de la sûreté publique, de l'ordre public, ou pour protéger la santé ou la moralité publiques ou les droits et les libertés d'autrui.

Cependant, la Constitution en son article. 37 indique : « L'Etat garantit la liberté d'association. Les pouvoirs publics collaborent avec les associations qui contribuent au développement social, économique, intellectuel, moral et spirituel des populations et à l'éducation des citoyennes et des citoyens (...)

Les modalités d'exercice de la liberté d'association varient en fonction du type d'association dont il est question. Une distinction existe notamment entre les associations à but lucratif, sans but lucratif (ASBL) et celles à des fins politiques. Particulièrement important dans le contexte des libertés publiques sont les deux derniers. La loi 004/2001 du 20 juillet 2001 portant dispositions applicables aux associations sans but lucratif (ABSL) et aux établissements d'utilité publique.

\section{c. Protection de la liberté d'association en RDC}

Tout groupe de personnes peut constituer une association. Cependant pour que celle-ci ait une existence juridique, bénéficie des avantages accordés par l'Etat et fonctionne selon les objectifs que ses créateurs lui ont assignés et conformément au respect des droits d'autrui, de l'ordre public et des bonnes mœurs, un certain nombre de formalités et de règles ont été énoncées. 
La constitution et le fonctionnement d'une association appartiennent à certaines règles. La participation d'un individu à une association est, en revanche, totalement libre et n'obéit à aucune règle. Le corollaire135 logique de la liberté d'appartenir à une association est celle de ne pas appartenir et aussi de se retirer (article 12).

Il existe des associations à but lucratif (sociétés civiles et commerciales) et sans but lucratif. La loi 004/2001 du 20 juillet 2001 concerne les associations sans but lucratif. Ce sont celles qui nous intéressent dans le cadre de l'étude des libertés publiques en son Article $1^{\mathrm{er}}$ : « L'Association sans but lucratif est celle qui ne se livre pas à des opérations industrielles ou commerciales, si ce n'est à titre accessoire, et qui ne cherche pas à procurer à ses membres un gain matériel. L'association sans but lucratif est apolitique. »

Au terme de l'Article 2: "L'Association sans but lucratif est de par sa nature et son objet soit :-Une association à caractère culturel, social ou éducatif ou économique;-Une organisation non gouvernementale $\mathrm{ONG}$, en sigle;

-Une association confessionnelle »

-La loi définit une catégorie particulière d'association qui connait un important développement en RDC : les organisations non gouvernementales (ONG) : En effet, l'article 35 : Est réputée organisation non gouvernementale, «ONG » en sigle, l'association sans but lucratif dotée de la personnalité juridique dont l'objet concourt au développement social, culturel et économique des communautés locales »

\section{Les conditions de constitution d'une association}

La loi définit les conditions de constitution d'une association qui comprennent : les conditions d'obtention de la personnalité juridique (Art 3 à 6). Pour pouvoir agir, une association doit être dotée de la personnalité juridique. Elle doit déposer une requête au ministère de la justice qui comprend essentiellement la liste des membres de la direction et de l'administration.

Les statuts de l'association sans but lucratif ne peuvent contenir aucune disposition contraire aux lois, aux bonnes mœurs ou à l'ordre public et doivent contenir un certain nombre de mentions destinées à l'identifier.

Concernant les règles de fonctionnement de l'association (Art10, 13,17et 19),les décisions de l'association sont prises par la majorité de ses membres et l'association ne peut avoir d'autre propriété que celle des immeubles nécessaires à la réalisation de son objet social. 137

S'agissant des mesures de dissolution ou de suspension, c'est le tribunal de grande instance qui peut prononcer la dissolution de l'association qui contrevient à ses statuts, à la loi, à l'ordre public ou aux bonnes mœurs (article 20). Le ministre de la justice ou le gouverneur de la province peut suspendre pour une durée ne dépassant pas trois mois les activités d'une ASBL ayant troublé l'ordre public ou porté atteinte aux bonnes mœurs. 
Les mesures de dissolution d'une association ou de suspension supérieure à trois mois sont de la compétence des autorités judiciaires gardiennes des libertés individuelles et non des autorités publiques.

Les obligations de l'Etat vis-à-vis des associations.

-Les associations sont considérées par la loi comme des partenaires de développement pour l'Etat : il est donc fait obligation à l'Etat de les associer « à la conception et à la réalisation de sa politique de développement au niveau local, provincial et national »(article 38)

-L'Etat doit faciliter l'existence et le développement des associations.

Les associations doivent bénéficier d'avantages fiscaux, d'exemptions de certaines taxes et de certaines formalités ainsi que du droit d'utilisation d'équipement et de fréquences radios (article 39)

-L'Etat a l'interdiction de faire immixtion dans la gestion des associations (art 40)

\section{Controverses en cas de violation de la liberté d'association}

Sur le terrain de la pratique, la réalisation de certaines conditions liées à la fondation des associations est devenue un obstacle sérieux à l'exercice du droit lui-même. Il faut relever les difficultés rencontrées par la population dans La constitution des dossiers, particulièrement en ce qui concerne l'authentification des statuts ainsi que l'obtention des certificats de bonne vie et mœurs :

-L'éclatement de la fonction notariale en circonscriptions amorcé à Kinshasa et qui se poursuivra sur l'ensemble du pays constitue une des voies possibles de sortie. Il en est de même du transfert de compétence pour ce qui est de l'autorité compétente pour accorder la personnalité civile aux associations, au regard de l'immensité du territoire national et de la compétence concurrente entre le pouvoir central et les provinces touchant la promotion et la sauvegarde des droits humains et des libertés fondamentales.

Il existe également des pratiques arbitraires de dissolution ou suspension des associations existantes sous prétexte du non respect des procédures légales. La corruption est également une entrave au libre déroulement des procédures administratives de déclaration.1

\section{La Liberté syndicale}

\section{II.1. Droits humains et liberté syndicale}

La liberté syndicale est la faculté reconnue aux travailleurs de constituer des associations spécifiques, dénommées syndicats, consacrées à la défense collective de leurs intérêts professionnels.

La Liberté syndicale déborde du cadre de la liberté d'association car elle est fondée plus largement sur le droit reconnu aux travailleurs de s'organiser par tous moyens ou défendre le droit au travail qui est également un droit spécifique reconnu et surtout le droit à des conditions de travail décentes et respectueuses des droits humains. Un des moyens les plus 
importants, qui est également consacré de façon autonome dans les textes nationaux et internationaux est le droit de grève.

Il n'existe pas de liberté syndicale sans pluralisme syndical, l'institution d'un syndicat unique qui monopolise la parole de l'ensemble des travailleurs est contraire à la démocratie et constitue une négation de la liberté syndicale.

\section{II.2. cadre juridique national et international de la liberté syndicale}

Le cadre international est le résultat du pacte international relatif aux droits sociaux, économiques et culturels qui aux termes des articles 8 et suivants dispose que« l'Etat partie au traité assure :

a) Le droit qu'a toute personne de former avec d'autres syndicats et de s'affilier au syndicat de son choix, sous la seule réserve des règles fixées par l'organisation intéressée, en vue de favoriser et de protéger ses intérêts économiques et sociaux.

L'exercice de ce droit ne peut faire l'objet que des seules restrictions prévues par la loi et qui constituent des mesures nécessaires, dans une société démocratique, dans l'intérêt de la sécurité nationale ou de l'ordre public, ou pour protéger les droits et les libertés d'autrui.

b) Le droit qu'ont les syndicats de former des fédérations ou des confédérations nationales et le droit qu'ont celles-ci de former des organisations syndicales internationales ou de s'y affilier.

c) Le droit qu'ont les syndicats d'exercer librement leur activité, sans limitations autres que celles qui sont prévues par la loi et qui constituent des mesures nécessaires, dans une société démocratique, dans l'intérêt de la sécurité nationale ou de l'ordre public, ou pour protéger les droits et les libertés d'autrui.

d) Le droit de grève, exercé conformément aux lois de chaque pays (...)

-Organisation Mondiale du Travail (OMT)

Le bureau de l'OMT a publié une série de lignes directrices portant sur le droit d'association des travailleurs. Le texte le plus pertinent dans le contexte de ce guide est la Convention sur le droit d'organisation et de négociation collective de 1949, ratifiée également par la RDC en 1969.

S'agissant de l'ordre juridique interne, la Constitution du 18 février en son article 38 : «La liberté syndicale est reconnue et garantie. Tous les Congolais ont le droit de fonder des syndicats ou de s'y affilier librement, dans les conditions fixées par la loi.

En effet, l'article 39 dispose que le droit de grève est reconnu et garanti; il s'exerce dans les conditions fixées par la loi qui peut en interdire ou en limiter l'exercice dans les domaines de la défense nationale et de la sécurité ou pour toute activité ou tout service public d'intérêt vital pour la nation.

La loi $n^{\circ} 015 / 2002$ du 16 octobre 2002 portant code du travail en Article 230 indique que « Les travailleurs et les employeurs... ont le droit de se constituer en organisations 
ayant exclusivement pour objet l'étude, la défense et le développement de leurs intérêts professionnels ainsi que le progrès social, économique et moral de leurs membres ».

-Un régime d'enregistrement et non d'autorisation préalable. La constitution d'un syndicat n'est soumise à aucune autorisation préalable de l'administration, ni de l'employeur (art. $231 \mathrm{du}$ Code de Travail), mais toutes les autres obligations et droits applicables aux associations de manière large se trouve mutatis mutandis applicable en matière des syndicats (articles 231-254 du Code de Travail).

Les formalités sont réduites à un enregistrement au ministère du travail sur le registre des syndicats :

La demande d'enregistrement :

-La demande d'enregistrement doit comporter l'identité des membres de la direction et de l'administration, les statuts de l'organisation. Les statuts doivent comporter la dénomination du syndicat, son objet, les conditions d'affiliation et de désaffiliation, le mode de nomination des membres de l'administration et de la direction et la durée de leur mandat, les règles de gestion financière, la procédure de modification des statuts ou de dissolution du syndicat (art 239).

-Les personnes chargées de l'administration et de la direction d'un syndicat doivent posséder la nationalité congolaise et être âgées de 21 ans au moins et doivent présenter certaines conditions de moralité et notamment concernant le casier judiciaire (art 241).

-La vérification faite par le ministère du travail tient à la légalité de l'objet du syndicat et la conformité des statuts à la législation en vigueur et aux règles concernant les membres de l'administration ou de la direction. Sous réserve de cette conformité le ministre du travail accorde l'enregistrement. Le refus d'enregistrement donne lieu à recours en justice (art 242 à 244).

Le syndicat jouit de la personnalité juridique qui lui permet en particulier d'acquérir les biens nécessaires à son fonctionnement et d'agir en justice. Ses biens ne peuvent être saisis (art 249).

La dissolution ne peut être décidée que par le syndicat lui-même en application de ses propres statuts. La dissolution ou la suspension ne peut faire l'objet d'une décision administrative (Article251 et 252)

La protection de la liberté syndicale : l'interdiction de la discrimination basée sur l'affiliation syndicale

L'affiliation syndicale, la non affiliation syndicale, la participation à des activités syndicales ou l'exercice d'un mandat de représentation syndicale ne peuvent donner à lieu à quelque discrimination que ce soit, en particulier sur le plan de l'emploi. Ils ne peuvent constituer un motif de licenciement (art 62). 


\section{La guerre médiatique et défis de la liberté de presse}

Dans ce paragraphe, il s'agira de relever l'impact des médias sur le pouvoir d'une part et, par ailleurs l'attitude des professionnels de la presse à l'égard des hommes qui détiennent le pouvoir politique avec quelques cas d'illustration dans la province du Katanga en RDC,

\section{III.1. La guerre médiatique et défis de la presse en RDC}

Elle consiste à utiliser les sources d'informations pour influencer les opinions publiques ou faire valoir son point de vue en temps de guerre.Elle sévit en particulier sur internet, où l'information peut être plus facilement diffusée sans contrôle. Selon l'historien militaire Pierre Razoux, la première utilisation de la guerre médiatique remonterait à la Seconde Guerre mondiale. Dans le pacifique, des émissions de radio pro-japonaises mais anglophones étaient animées par des jeunes femmes surnommées rose de Tokyo ${ }^{13}$.

La défaite américaine au Viêt Nam serait en partie due aux médias qui ont influencé l'opinion publique américaine. Forts de cette expérience, durant la guerre du Golfe, les médias ont fait l'objet d'un contrôle accru de la part des autorités américaines. En ce qui concerne la guerre civile syrienne, les médias occidentaux imputent généralement et immédiatement la responsabilité des massacres aux forces loyalistes, en se basant sur les informations du controversé OSDH. Pourtant, des informations permettent d'envisager la responsabilité des rebelles.

Une intervention nécessaire qui respecte les principes de proportionnalité et de la progressivité permet d'éviter qu'une manifestation ne dégénère en violences, et particulièrement en violences commises par des policiers.

Cette situation est la preuve réelle de l'influence politique de ce qui opposa le député et gouverneur du Katanga Moise Katumbi Chapwe et Jean-Claude Muyambo ancien ministre des affaires sociales et député national en RDC.

Nous relèverons ici, primo, l'attitude des journalistes par rapport aux hommes au pouvoir, secundo, les enjeux de cette contradiction, tertio le rôle de la police dans sa mission de l'ordre public, quarto la décision du conseil audio visuel de la communication et quinto savoir la position de la justice a cet effet.

Pour rappel, les deux hommes sont d'une même souche tribale Bemba-sanga deux tribus sœurs dans le sud de la RDC et spécialement la province cuprifère et ont cependant œuvré ensemble comme des hommes d'affaires, ce qui n'étonnera personne en me lisant. Toutefois pour des intérêts divergents, à savoir, politiques, économiques et leurs relations particulières, ils sont en parfaite discordance. Mais une chose est sûre comme le dit un adage selon lequel « les lignes sales se lavent en famille » et nous nous permettons de s'arrêter par là pour raison de curiosité scientifique et on en passe!

13 The Legend of Tokyo Rose [archive] par Ann Elizabeth Pfau. 
La ville de Lubumbashi est en émoi. Des milices fidèles au gouverneur sortant Moïse Katumbi Chapwe et à Jean-Claude Muyambo Kyassa, ancien ministre, se sont affrontées mercredi 15 juin2011 dans la ville. Les deux personnalités appartiendraient à la mouvance kabiliste. Une interview très critique de Muyambo sur le bilan de Katumbi a servi de détonateur.

Des voix se sont élevées pour dénoncer le climat d'intolérance politique qui y règne autant que l'attitude ambiguë des forces de l'ordre. Celles-ci sont restées impassibles pendant que les partisans du gouverneur, les fameux "Cent pour cent", mettaient à sac le domicile de Muyambo qui a décidé de se mettre en porte-à-faux vis-à-vis de la justice congolaise. Il a poursuivi la publication de ses lettres ouvertes au gouverneur du Katanga.

Il en est à la 14ème alors que, à la 12ème, le Procureur général de la République (PGR), dans un communiqué, avait demandé que cessent les débats et autres commentaires dans les médias au sujet de la polémique qui les oppose; l'objectif étant de ne pas influencer l'enquête judiciaire ouverte à ce sujet par Parquet Général.

C'est pour cette même raison que le PGR, en accord avec le CSAC (Conseil supérieur de l'audiovisuel et de la communication), avait suspendu les chaines de télé Nyota et Jua, appartenant aux deux protagonistes. Que Jean-Claude Muyambo Kyassa poursuive la publication de ses lettres ouvertes à Moïse Katumbi ne pourrait être interprété autrement que comme un défi lancé au parquet général de la République et une exhibition de la jouissance d'une impunité qui ne dit pas son nom ${ }^{14}$.

Jean-Claude Muyambo Kyassa, président de la "Solidarité congolaise pour la démocratie et le développement" (SCODE).

Que s'est-il passé à Lubumbashi le mercredi 15 juin 2011? Selon des sources concordantes, tout a commencé le mardi 14 juin dans la soirée. La "RTLJ" (Radio Télévision Lubumbashi Jua), un média privé appartenant à Jean-Claude Muyambo, président du parti "Solidarité congolaise pour la démocratie et le développement" (Scode), a diffusé des larges extraits d'une interview que ce dernier venait d'accorder au cinéaste et journaliste belge Thierry Michel. Dans cet entretien, Muyambo s'est livré à un véritable réquisitoire sur le bilan du gouverneur sortant Moïse Katumbi à la tête du Katanga.

Mercredi 15 juin, des individus identifiés comme étant des supporters du Tout-Puissant Mazembe, familièrement appelés «Cent pour cent», ont attaqué le domicile de Muyambo.

C'est en ce lieu que sont logées les installations de "RTLJ". "Brandissant des calicots diffusant des messages obscènes, des menaces de mort et scandant des chansons de guerre, ces manifestants ont cassé les vitres des bureaux de la chaîne RTLJ, incendié des véhicules qui s'y trouvaient, et emporté certains effets de valeur, indique un communiqué de l'Asadho-Katanga daté du 16 juin. L'incendie des installations de ladite chaîne a été empêché de justesse par l'intervention de la police nationale congolaise. Néanmoins, il est enregistré un bilan de 15 blessés graves dont 7 journalistes et agents administratifs de la chaîne.”

Qui a transmis les images querellées à la «RTLJ»?

14 Kinshasa, 22/11/2012 / Politique. 
Dans un communiqué daté du vendredi 17 juin intitulé «A propos des évènements de Lubumbashi de ce mercredi 15 juin 2011», Thierry Michel - qui reconnaît avoir réalisé une interview avec Muyambo - pointe un doigt accusateur en direction d'un caméraman de la RTLJ non autrement identifié.

«Sans m’en avertir, écrit-il, un caméraman de la chaîne de télévision Jua, appartenant à Maître Muyambo, a filmé dans mon dos et à mon insu cet entretien. Cet enregistrement a ensuite été copié et transmis à Moise Katumbi, cette fois-ci à l'insu de Maître Muyambo.» Et d'ajouter : «Moise Katumbi, au vu des critiques sur la gestion de la province émises par son concurrent au sein de la même famille politique, a alors décidé de faire diffuser cette interview sur la plupart des chaînes privées» et sur la station régionale de la RTNC (Radio télévision nationale congolaise).

Pour la petite histoire, Muyambo et Katumbi appartiennent à la Majorité présidentielle tout étant des adversaires politiques au niveau provincial. Le cinéaste belge de jurer ses grands dieux que «cet entretien n'était absolument pas destiné à être diffusé en RDC dans la période actuelle et encore moins dans son intégralité.» Selon lui, «cette diffusion a résulté d'un choix délibéré des acteurs politiques katangais». Il s'estime par conséquent «avoir été utilisé, instrumentalité par les uns et les autres à des fins politiciennes et partisanes de politique locale».

Réagissant à ces événements, la Société civile du Katanga a donné de la voix en fustigeant la montée de «l'intolérance politique» dans la province du Katanga et la «passivité» de la police nationale. Dans un communiqué daté du vendredi 17 juin, elle fustige la «complicité» des autorités urbaines de Lubumbashi «dans l'organisation de la manifestation illégale ayant abouti aux actes de vandalisme.»

Cette entreprise criminelle, poursuit-elle, a bénéficié d'un concours indispensable des agents de la police nationale congolaise qui ont assisté passivement et des agents de la brigade urbaine d'assainissement.» Et de relever qu'«en cette période pré-électorale, il est constaté dans la province du Katanga, une escalade de violence due principalement par la tenue des discours d'intolérance de la part de certains opérateurs politiques et de l'entretien par eux des milices droguées et prêtes à défier l'Etat Congolais.» La Société civile se dit par ailleurs préoccupée de constater «qu'aucune autorité nationale et provinciale ne se soit prononcée pour condamner et décourager ces actes d'intolérance politique qui surviennent dans un contexte électoral déjà tendu et qu'eux-mêmes font partie de la famille politique qui soutient la candidature de Monsieur Joseph Kabila aux présidentielles de septembre $2011^{15}$.»

Le Conseil Supérieur de l'Audio-visuel et Communication avait décidé de suspendre la Radio Télévision Lubumbashi Jua : ce dit-on

15 B.A.W;Ci-après le texte intégral du communiqué de la Société civile du Katanga :Communiqué conjoint de la société civile du Katanga,Les installations de la Radio Télévision «Jua» paient les frais de l'intolérance des acteurs politiques du Katanga. 
L'article $11 \mathrm{du}$ Code de déontologie et de l'éthique journalistique du journaliste congolais affirme le devoir de « respecter la dignité humaine, la vie privée, la sphère d'intimité des individus, ainsi que celles des institutions et autorités publiques, l'ordre public et les bonnes mœurs ». De manière générale la Loi de 1996_indique qu' " au risque de tomber sous le coup de la loi, il est interdit:

a) de publier les actes d'accusation et tous autres actes de procédure judiciaire avant qu'ils n'aient été lus en audience publique;

b) de divulguer les délibérations des cours et tribunaux. Il en est de même des informations sur les travaux et les délibérations du Conseil Supérieur de la Magistrature sans l'autorisation du conseil lui-même;

c) de reproduire en photographies, dessins ou portraits de tout ou partie des circonstances des crimes de sang, des crimes ou délits touchant aux mœurs, sauf demande expresse du chef de la juridiction saisie du cas.

Cette interdiction s'applique également à toute illustration concernant le suicide des mineurs, sauf autorisation écrite du procureur de la République;

d) d'enregistrer, de fixer ou de transmettre la parole ou l'image aux audiences des cours et tribunaux, sauf autorisation du chef de la juridiction.

Il en est de même pour les procès en diffamation lorsque les faits incriminés concernent la vie privée des personnes;

e) de publier ou de diffuser des informations sur un viol ou sur un attentat à la pudeur en mentionnant le nom de la victime ou en faisant état des renseignements pouvant permettre son identification, à moins que la victime n'ait donné son accord écrit;

f) d'ouvrir ou d'annoncer publiquement des souscriptions ayant pour objet de payer des amendes, frais et dommages et intérêts prononcés par des condamnations judiciaires sous peine de poursuites "».

En respect de la vie privée et de l'honneur des personnes, plusieurs pays, dont le nôtre, répriment les enregistrements ou les transmissions d'images des personnes dans/ou à partir des lieux privés sans le consentement de celles-ci. Avec un peu plus de rigueur, la Loi française sur la presse affirme que les précautions à charge des professionnels des médias et de toutes personnes s'appliquent aussi aux messages SMS diffusés à l'antenne et aux sites internet des chaînes.

L'article 2 du Code de déontologie et d'éthique du journaliste congolais demande au journaliste de : « faire preuve, dans ses tâches quotidiennes, d'équité, d'exactitude, d'honnêteté, du sens de responsabilité, d'indépendance et de décence dans la relation des faits liés aux individus et à la société ».

L'article 6 du même Code ajoute que le journaliste doit « rechercher à tout instant le triomphe de la vérité, par une relation exacte, honnête, fidèle et loyale des faits dûment avérés et vérifiés et des informations obtenues sans chantage et sans surprendre la bonne foi de quiconque ».

Les émissions de la Radio Télévision Lubumbashi Jua (RTLJ) ont été suspendues, vendredi 13 janvier, pour une durée de sept jours par le Conseil supérieur de l'audio-visuel et 
de la communication (CSAC)/Katanga, sur instruction de la direction nationale. Le CSAC reproche à cette chaîne appartenant à Muyambo Kyassa la diffusion des propos méprisants et autres insinuations injurieuses à l'endroit de certaines personnalités du pays.

Le signal de la Radio Télévision Lubumbashi Jua a été coupé vers 12 h 15, heure locale. Le président du CSAC au Katanga venait de remettre aux responsables de la RTLJ la notification de la décision de suspension des émissions. A la RTLJ, l'on dit s'incliner devant cette décision. Mais, Jean-Claude Muyambo a déploré le fait que, sur d'autres médias, des journalistes s'attaquent à lui, sans qu'il y ait des réactions de la part du CSAC. Le directeur général de la RTLJ, Guelord MUKANYA a déclaré «dernièrement, à Kinshasa, nous avons suivi quelqu'un qui a publié [les résultats], avant même la CENI, indiquant que Muyambo était déjà un candidat malheureux. Cela n'a pas inquiété le CSAC et ça n'a pas posé problème. Ici, à Lubumbashi, personnellement, j'ai suivi comment on l'insultait.»

Le président provincial du CSAC, Richard KALUMBA, a jugé cette revendication fondée. "A travers la décision de vendredi, le CSAC met aussi en garde tout journaliste ou autre personne qui utiliserait les medias pour s'attaquer aux autres», a-t-il précisé.

$\mathrm{Au}$ cours d'une page magazine spéciale, diffusée le 11 janvier et rediffusée plusieurs fois le lendemain, Jean- Claude Muyambo s'est attaqué à certaines personnalités. Pour le CSAC, ce dernier a utilisé des «propos méprisants et des insinuations injurieuses ${ }^{16}$ »

III.2. Appréciation critique : défis et effectivité de la liberté de réunion et de manifestation.

Une intervention nécessaire qui respecte les principes de proportionnalité et de la progressivité permet d'éviter qu'une manifestation ne dégénère en violences, et particulièrement en violences commises par des policiers.

De ce qui précède, il convient de relever que ni les instances judiciaires, ni le conseil de l'audio-visuel de la communication de la république étaient dans l'incapacité de résoudre ces défis au regard des faits énoncés dans le paragraphe précédent, ce qui explique mieux l'influence des hommes au pouvoir dans la presse d'une part et d'autres part la subordination de l'appareil législatif au pouvoir législatif : « le pouvoir a-t-il raison d'arrêter le pouvoir selon MONTESQUIEU.

En voici quelques exemples similaires :

-Le 7 septembre 2011 le gouverneur provincial de Kinshasa, a interdit toute manifestation à caractère politique (marche ou sit-in) sur la voie publique jusqu'au 11 septembre 2011 pendant la période consacrée aux opérations de dépôt des candidatures de la Commission Electorale Nationale Indépendante.

Cette interdiction limitée dans le temps, concernant l'ensemble du monde politique, est intervenue suite à des violents affrontements entre les militants de la majorité présidentielle et de l'Opposition et des forces de sécurité, entraînant aussi la mort d'un partisan de l'oppo-

16 Cet article a été écrit par Odilon et posté le 16/01/2012 à 19:44 et est classé dans Provinciale. Bookmaker le permalien. Suivez les commentaires via le flux RSS de cet article. 
sition politique. Le but de cette mesure était de permettre à ces opérations de se dérouler dans la sérénité afin de favoriser un climat social apaisé au cours du processus électoral.

-En janvier-février 2007 puis en février-mars 2008, les forces de sécurité ont fait usage de la force à l'encontre des membres du groupe Bundu Dia Kongo de façon disproportionnée dans plusieurs endroits du Bas-Congo. Selon les chiffres de la communauté internationale plus qu'une centaine de manifestants ont été tués ainsi qu'une dizaine de membres de la Police. Les Nations-Unies ont condamné par l'usage disproportionné de la force par la Police

-La marche de Jean Pierre Bemba : le 27 juillet 2006, revenant d'une tournée électorale, cet homme politique, arrivé à l'aéroport de N'Djili, est descendu vers la ville au pas. Une marche de trois heures a eu lieu de 500000 et un million de personnes qui ont accompagné le leader fondateur du Mouvement de libération du Congo (MLC) jusqu'au stade Tata-Raphaël pour son dernier meeting de campagne avant les élections présidentielles. Sur le trajet de la manifestation, les foules non contrôlées ont commis toutes sortes d'exactions et de violences graves sur la population habitante des quartiers traversés. La PNC a été débordée ce jour-là et incapable de remplir sa mission principale de protection et de maintien de l'ordre public. 1

Le Procureur général de la République aura-t-il échoué à dissiper la virulente et nocive guerre médiatique que se mènent le Gouverneur du Katanga et son adversaire politique Jean-Claude Muyambo? Le dernier cité paraît défier l'autorité judiciaire en poursuivant la publication pourtant interdite de ses lettres ouvertes contre le $n^{\circ} 1$ de la province cuprifère.

\section{RECOMMANDATIONS}

Les conclusions sur le droit de manifester, de se réunir ou se constituer en syndicats, d'intervenir pour éviter le débordement voir la perturbation ou le trouble de l'ordre public ou de diffuser les informations en RDC appellent quelques recommandations :

- que la police soit celle qui intervient non seulement pour disperser les gens à la solde des autorités mais aussi une police bien formée, intégrée et non un ensemble des individus analphabètes avec pour objectif la recherche de la survie au quotidien.

- que les réunions ou manifestations publiques soient diligentées par les autorités en vue de faire respecter la liberté d'opinion ou d'expression.

- que la profession journalistique soit neutre, juste en respectant le sens de la mesure et non poursuivre les attitudes des dirigeants politiques à cause de leurs intérêts.

- que les chaînes de radiotélévision soient régies par des lois strictes et non les laisser semer la haine des protagonistes politiques.

\section{CONCLUSION}

La plus large liberté d'expression est essentiellement pour mettre en œuvre la responsabilité politique des gouvernants, corolaire de la souveraineté populaire. L'article 19 de la déclara- 
tion universelle de droits de l'homme dispose que « Tout individu a droit à la liberté d'opinion et d'expression, ce qui implique le droit de ne pas être inquiété pour ses opinions et celui de chercher, de recevoir et de reprendre, sans considération de frontières, les informations et les idées par quelque moyen d'expression que ce sort »

Une exigence d'un large débat public que consacre le premier amendement de la constitution américaine pris selon la cour suprême des États-Unis repose sur les principes que la diffusion de l'information la plus large possible à partir des sources diversifiées et antagonistes est essentiel pour le bien-être public qu'une presse libre est une condition de société libre.

Dans son allocution qu'il prononça à l'université de Nairobi sur « le rôle de droit et le développement économique » l'avocat Jean Salem KAPYA KABESA avait martelé que « selon les époques, le temps et l'histoire des hommes et selon les réalités appropriées de chaque Etat en quête de la modernité, la décentralisation que ça soit au Rwanda, Burundi, RDC, Tanzanie, Kenya et partout ailleurs en Afrique, apparaît comme un véhicule à double vitesse dont ses leviers sont l'économie et le développement; or cela n'est possible que par le travail bien fait, ceci engendre la croissance, celle-ci le développement économique ${ }^{17}$...Il conclut en citant un écrivain du $18^{\text {ème }}$ siècle, Odilon Barrot, selon qui « c'est le même marteau qui frappe, mais on raccourcit le manche ».

La liberté des réunions pacifiques et la liberté de manifestation sont des droits d'ordre constitutionnel, sanctionnés par les traités internationaux. Comme pour toutes les libertés publiques, les seules restrictions à leur exercice ne peuvent être tirées que des atteintes aux droits d'autrui, à l'ordre public et aux bonnes mœurs tel qu'énoncées par la loi (article 25 Constitution, art. 1 Décret-loi 1999).

Comme c'est le cas pour les autres libertés publiques, la notion d'ordre public doit être strictement interprétée, le risque de trouble invoqué devant être sérieux et caractérisé. L'interprétation extensive de cette notion permet souvent aux autorités publiques, souvent sous couvert de sécurité publique, à commettre de graves violations de ces libertés.

Avant tout usage d'armes à feu, cette autorité fait trois sommations formulées à haute et intelligible voix dans les termes suivants : « obéissance à la loi; on va faire usage d'armes à feu; que les bons citoyens se retirent ».

Ces principes sont d'une très grande importance. Ils permettent aux responsables de prendre les mesures strictement nécessaires au maintien de l'ordre tout en assurant aux citoyens le respect de leurs libertés fondamentales. Une intervention nécessaire qui respecte les principes de proportionnalité et de la progressivité permet d'éviter qu'une manifestation ne dégénère en violences, et particulièrement en violences commises par des policiers ...

Il est regrettable que ni les instances judiciaires, ni le conseil de l'audio-visuel et communication n'ont pas réussi de sanctionner à l'égard des uns et des autres les atteintes à la

17 Allocution prononcé par maitre KAPYA KABESA à la deuxième conférence régionale sur « le rôle du droit et le développement économique » organisée par la fondation Konrad Adnauer Stiftung du 01 au 04 novembre 2012. 
liberté individuelle, les violences de leur camps respectifs entre les deux hommes, ni la police dans sa mission de maintenir l'ordre public n'a réussi à défier cette aventure. Ce qui nous conduit à nous demander pourquoi et comment le respect des libertés publiques doit et sera respectées pour que l'état de droit trouve sa valeur intrinsèque.

\section{BIBLIOGRAPHIE}

1. Déclaration des droits de l'homme et du citoyen du 26 août 1789.

2. loi ${ }^{\circ} 015 / 2002$ du 16 octobre 2002 portant code du travail

3. Constitution La RDC du 18 février 2006

4. Convention sur le droit d'organisation et de négociation collective de 1949, ratifiée également par la RDC en 1969

5. Décret $n^{\circ} 08 / 23$ du 24 septembre 2008 portant organisation et fonctionnement de l'Inspection Générale d'Audit.

6. Décret-loi 196 du 29 janvier 1999 portant réglementation des manifestations et réunions publiques

7. loi 004/2001 du 20 juillet 2001 concerne les associations sans but lucratif.

8. loi électorale de 2006 et une circulaire du Ministre de l'Intérieur (002/2006 du 29 juin 2006)

9. Loi organique du 10 août 2011

10. Loi organique $\mathrm{n}^{\circ} 2009-257$ du 5 mars 2009 relative à la nomination des présidents des sociétés France Télévisions et Radio France et de la société chargée de l'audiovisuel extérieur de la France.

11. Pacte international relatif aux droits sociaux, économiques et culturels

12. résolution 34/169 des Nations Unies adoptée en 1990 à la Havane.

13. Allocution prononcée par Maître KAPYA KABESA à la deuxième Conférence régionale sur « le rôle du droit et le développement économique » organisée par la fondation Konrad Adenauer Stiftung du 01 au 04 novembre 2012 à Nairobi.

14. BORIS LIBOIS, Éthique de l'information, essai sur la déontologie journalistique, Ed. Presse Universitaire de Bruxelles, 1994

15. CHARVIN, R, et SUEUR, J-J, Droits de l'homme et libertés de la personne, Litec, 5éme éd, 2007.

16. Cynthia Fleury, "La Fin du courage", Paris, Fayard, 2010).

17. DE TOCQUEVILlE A. De la démocratie en Amérique, éd. Union générale, Paris 1990

18. KAPYA KABESA Jean Salem Marcel, les défis de la liberté de presse en RDC (enjeux pour la ville de Lubumbashi,in annales de Kolwezi, n², juillet 2009,pp.34-47

19. Vatican II décret sur les moyens de communication, n5 